\section{OP-48 MOTHERS' PERCEPTION ABOUT DETERMINANTS OF CHILD HEALTH: PHOTO VOICE EXPLORATION IN THE SUNDARBANS, WEST BENGAL}

Shibaji Bose, Upasona Ghosh, Rittika Bramhachari, Sabyasachi Mondol. IIHMR Institute of Health Management Research, Kolkata (West Bengal), India

\subsection{6/bmjgh-2016-EPHPabstracts.48}

Background Child health status in the Indian Sundarbans - the delta region in the southeast part of West Bengal - is characterized by chronic malnutrition and high prevalence of communicable diseases, and greatly determined by socio-economic and political factors. Mothers' perception regarding determinants of child health is pertinent to strengthen community level decisionmaking on health service delivery. In the present study we used 'photo voice', a visual action research technique, to glean the perception of the mothers on their children's health and its determining factors.

Methods For the present study, the photo voice technique was conducted in three randomly selected blocks of the Indian Sundarbans, with three groups each (average 8-10 members), the members having at least one child in the age range 0-6 years. Training about the technical process of photo documentation was conducted with each group prior to the research. Respondents took two rounds of photographs within six months, interspersed by fortnightly group meets with researchers as facilitators. The research findings were later communicated to different community policy stakeholders during policy interface sessions.

Findings Respondents stressed on specific health issues - disabilities, mental health and non-communicable diseases of their children - that they could manifest through photo, other than documenting common and frequently occurring communicable diseases. While reflecting on the determinants, respondents' photos and narratives focused on determinants of child health which they prioritise on the basis of lived experiences. The determinants that figured prominently included: (1) food insecurity and related malnutrition among the children; (2) livelihood; (3) shelter; (4) water and sanitation; (5) poor healthcare services; (6) accessibility issues; (7) general health and hygiene awareness; and (8) climate. During the group meets, respondents also stressed on social factors not captured trough photo, including early marriage, recurrent pregnancies and domestic violence.
While selecting the priority issues to be presented in the interfaces with community policymakers, respondents selected five issues: (1) malnutrition; (2) livelihood; (3) water and sanitation hygiene; (4) accessibility; and (5) rupture of embankments. During interfaces with local non-government organisations, key community leaders and local health providers, respondents stressed on these determinants, which according to them are intertwined and should be considered in conjunction for improving the health of their children. Respondents' interface with community policymakers resulted in initiation of road infrastructure, human resource availability in the primary health centres and local government representatives raising related demands at the key forum and committees dedicated to planning on convergence.

Discussion Photo voice has the potential in voicing community's knowledge regarding the determinants of health of their children through the eyes of the mothers. Our study explored the perceived health determinants of geo-climatically vulnerable population of the Sundarbans. The study adds to the existing knowledge of difference in perception of policymakers and implementers of the health system, who have a tendency to compartmentalise issues in terms of health and non-health spheres on the one hand, and the community members viewing health of their children as an integrated phenomenon on the other hand.

Our study explored the social determinants of child health through a feminist lens, a major theoretical underpinning of photo voice as a community-based action research method. It also seeked to voice vulnerable populations' knowledge grounded in experience and to recognize local expertise, an insight that cannot be fully realised from the outside, as against the sectoral perceptions of the largely masculine power and predominantly male bastion of decision-makers in most policy bodies. Our study thus adds to existing literature of how participatory action research techniques like community-owned documentary photography and narratives will empower vulnerable populations to record community health as an integration of medical and socio-political factors to inform basic health policy.

Grant funding (DFID - Department for International Development, UK) for research but no other competing interests. 